
allemande

48-2 | 2016

Les espaces publics des pays germanophones, des espaces publics transnationaux?

\title{
Mariage, famille et sexualité vus par les féministes radicales dans l'Allemagne wilhelmienne (1890-1918)
}

\section{Anne-Laure Briatte-Peters}

\section{(2) OpenEdition}

\section{Journals}

Édition électronique

URL : https://journals.openedition.org/allemagne/406

DOI : 10.4000/allemagne.406

ISSN : 2605-7913

Éditeur

Société d'études allemandes

Édition imprimée

Date de publication : 28 décembre 2016

Pagination : 427-437

ISSN : 0035-0974

Référence électronique

Anne-Laure Briatte-Peters, « Mariage, famille et sexualité vus par les féministes radicales dans

I'Allemagne wilhelmienne (1890-1918) », Revue d'Allemagne et des pays de langue allemande [En ligne],

48-2 | 2016, mis en ligne le 28 décembre 2017, consulté le 19 mai 2021. URL : http://

journals.openedition.org/allemagne/406 ; DOI : https://doi.org/10.4000/allemagne.406 


\section{Mariage, famille et sexualité vus par les féministes radicales dans l'Allemagne wilhelmienne (1890-1918)}

\section{- Anne-Laure Briatte-Peters*}

Laile radicale du mouvement féministe bourgeois a vu le jour, dans l'Allemagne impériale, au début des années 1890, et a cessé d'exister en tant que telle au lendemain de la Première Guerre mondiale. Minoritaire dans le mouvement de femmes bourgeois, elle se distinguait par sa détermination dans ses revendications de l'égalité de droits pour les femmes dans tous les domaines de l'existence: le droit de faire des études et d'exercer des métiers qualifiés, de décider elles-mêmes de ce à quoi elles emploieront leurs talents, de participer aux processus de décision relatifs à la vie en société et dans l'État, ainsi que, à cette fin, le droit de vote.

On pourrait penser que les féministes radicales, qui accordaient une valeur toute particulière à l'accès des femmes à l'espace public, n'étaient pas particulièrement intéressées par la sphère privée de la domesticité. Pourtant, à y regarder de plus près, il apparaît que la famille et la sphère dite privée constituaient un des thèmes centraux de leur mouvement et que, avec la lecture politique qui était la leur, elles voyaient là un enjeu central pour l'émancipation féminine. Dans ce qui suit, l'analyse des débats sur le mariage parmi les féministes allemandes vise à montrer que ces femmes avaient parfaitement saisi la nature politique de la subordination des femmes dans la famille et qu'elles ont introduit dans la sphère publique ce sujet apparemment privé, bien avant que les féministes des années 1960-1970 ne se ressaisissent de ce complexe thématique.

Partant du débat sur le mariage, particulièrement vif au tournant du siècle, cet article cherche à déterminer les positions des féministes radicales sur le mariage, la famille et la sexualité et à saisir leur lien avec leur conception de l'émancipation féminine. Il sera d'abord fait un retour sur le contexte dans lequel ce sujet a émergé dans la dernière décennie du XIX ${ }^{e}$ siècle, lorsque le caractère profondément inégal du code civil unifié (Bürgerliches Gesetzbuch, BGB), qui était sur le point d'être adopté, a suscité de vives réactions chez les féministes allemandes. Face à leur critique de l'institution du

\footnotetext{
* Maîtresse de conférences en études germaniques à l'Université Paris-Sorbonne.
} 
mariage telle qu'elle était codifiée dans le droit familial, et nous demandant comment cette critique se traduisait dans le comportement conjugal des féministes en question, nous examinerons ensuite les constellations familiales dans lesquelles elles vivaient. Il faudra finalement tenter d'identifier les raisons de l'opposition des féministes radicales à la morale et au code familial en vigueur afin de comprendre pourquoi, si ce n'est pas la recherche hédoniste du plaisir, des féministes radicales autour de 1900 s'efforçaient de faire valoir une nouvelle morale sexuelle et de nouveaux rapports entre les sexes.

\section{Le droit familial dans le code civil unifié comme justification d'un engagement féministe}

Avant que la création de l'Empire allemand ne réalise l'unification nationale en 1871, la Confédération germanique rassemblait quelque 35 États souverains ayant chacun son propre gouvernement, son parlement et ses lois. Le statut légal des femmes, codifié dans le droit privé, qui résultait par ailleurs d'une stratification du droit coutumier, de codifications locales et de l'application des lois, différait donc d'un État à l'autre. Il était complexe, non univoque et relevait de diverses traditions juridiques ${ }^{(1)}$, ce qui amène Ute Gerhard à le comparer à une mosaïque bigarrée (ein bunter Flickenteppich ${ }^{(2)}$ ) pour souligner la pluralité des statuts juridiques des femmes dans les États allemands avant l'unification du code civil.

\section{Une accentuation de la discrimination des femmes dans le $B G B$}

Bien que célébré par les experts juristes contemporains comme le «manifeste d'une culture juridique libérale ${ }^{(3)}$, le BGB constituait un pas en arrière pour le statut juridique des femmes, en particulier en comparaison avec le code civil prussien en vigueur depuis 1794, le Allgemeines Landrecht (ALR), qui était relativement libéral dans ses dispositions relatives au divorce et au droit des femmes à la propriété( ${ }^{(4)}$.

Les quatre articles et dispositions suivants du BGB en particulier ont suscité un vif sentiment d'injustice parmi les femmes:

- Le paragraphe dit «de l'obéissance» («Gehorsamparagraph»), qui donne au mari le pouvoir de décision dans tous les domaines relatifs à la vie commune des époux, notamment le lieu de résidence et l'habitation ( $\$ 1354$ BGB, 1.1.1900 $\left.{ }^{(5)}\right)$. Cette clause restreignait considérablement la compétence légale et le pouvoir d'action des femmes mariées.

- Certes, l'expression «autorité parentale» (\$1626 BGB) a supplanté celle d'«autorité paternelle», mais le droit familial, contenu dans le quatrième livre du BGB,

1 Ute Gerhard, "Grenzziehungen und Überschreitungen. Die Rechte der Frauen auf dem Weg in die politische Öffentlichkeit», in: ID. (éd.), Frauen in der Geschichte des Rechts: von der Frühen Neuzeit bis zur Gegenwart, Munich, Beck, 1997, p. 515-519.

2 Ute Gerhard, Gleichheit ohne Angleichung. Frauen im Recht, Munich, Beck (Beck'sche Reihe, 391), 1990, p. 145.

3 Cit. d'après U. Gerhard, Gleichheit (note 2), p. 119.

4 Ute Gerhard, «Le droit civil, un outil de domination masculine? », in : Encyclopédie pour une histoire nouvelle de l'Europe (en ligne), 2016, mis en ligne le 14.11.2016, consulté le 14.11.2016, ehne.fr/article/ genre-et-europe/le-droit-civil-un-outil-de-domination-masculine.

5 Tous les articles du BGB cités dans ce texte se réfèrent à la version du BGB entrée en vigueur le 1.1.1900. 
sanctionnait de facto l'autorité paternelle en ce qu'il accordait au père en dernière instance le droit et le devoir d'exercer l'autorité parentale (\$1627). La mère ne pouvait être amenée à exercer ce droit qu'en qualité de représentante du père, dans le cas où celui-ci était absent ou privé de droits civils.

- Le droit familial considérait le père d'enfants illégitimes comme étant sans lien de parenté avec eux, sauf dans le cas d'une reconnaissance paternelle, et n'obligeait le père biologique qu'à s'acquitter d'une pension alimentaire. Par ailleurs, l'exception plurium concubentium (\$1717 BGB), que peut opposer le défenseur qui soupçonne l'enfant naturel d'avoir été engendré par un autre que lui durant la période supposée de la conception, suffisait à priver l'enfant de tout droit vis-à-vis de son père biologique, et par suite à exempter le père de l'obligation alimentaire.

- Dans le code civil unifié, le mari jouissait du droit de gestion et d'usufruit sur les biens de sa femme ( $\$ 1363$ BGB). La femme mariée ne pouvait disposer que de l’argent qu’elle gagnait par sa propre activité (\$1367). Mais étant donné la médiocrité de la rémunération du travail féminin et le fait que le mari était en droit de résilier son contrat de travail à elle à tout moment et sans délai (\$1358), ce droit pour les femmes de gérer leur argent était d'une bien fragile constitution.

Dans ces quatre cas, les dispositions juridiques concernant le droit de propriété et d'action des femmes mariées entérinent la subordination des femmes dans le mariage et leur dépendance économique vis-à-vis de leur mari, dont le rôle ancestral de tuteur marital (Ehevormund ${ }^{(6)}$ ) était consolidé par le BGB.

\section{Ignorées}

Lorsque la grande entreprise d'unification du code civil a commencé dans l'Empire allemand en 1873, les membres de l'Association générale des femmes allemandes (Allgemeiner Deutscher Frauenverein, ADF) autour de Louise Otto-Peters (1819-1895), l'une des pionnières du féminisme allemand, à Leipzig y ont vu l'opportunité d'une nouvelle codification des droits civils pour les femmes. Ainsi, elles ont publié dès 1876 une brochure d'information sur les droits des femmes dans les lois allemandes et adressé une pétition aux législateurs, leur demandant de veiller au principe d'égalité et de prendre en considération les intérêts des femmes, en particulier dans les lois portant sur le mariage et sur la tutelle. Mais leurs demandes, pourtant très explicites, ont été ignorées, et le BGB allait être discuté en deuxième, puis en troisième lecture, pour être finalement adopté au parlement (Reichstag) en 1896.

Entre-temps, au début des années 1890, une poignée d'Allemandes, parmi lesquelles Anita Augspurg (1857-1943), Marie Stritt (1855-1928) et Marie Raschke (1850-1935), avaient fait des études de droit: la première à l'université de Zurich, étant donné qu'il n'était pas encore permis à une femme de s'inscrire dans une université en Allemagne, la seconde en autodidacte, et la troisième en autodidacte puis à l'université, de Berlin en auditrice libre puis de Berne pour son doctorat (1899). Fortes de ce savoir juridique, pour Anita Augspurg même attesté par l'obtention du grade de docteur (Dr. jur.) en 1897, elles ont soumis des amendements à la version en lecture du BGB pour une plus grande égalité entre les sexes dans le droit familial.

6 Stephan Buchiolz, "Das Bürgerliche Gesetzbuch und die Frauen: zur Kritik des Ehegüterrechts», in: U. Gerhard (éd.), Frauen (note 1), p. 675. 
Elles et leurs compagnes de lutte dans le mouvement féministe ont cherché à mobiliser les Allemandes en grand nombre. Au milieu des années 1890, ce qui était en train de devenir l'aile radicale du mouvement féministe bourgeois, autour de Minna Cauer (1841-1922), Anita Augspurg, Lida Gustava Heymann (1868-1943) et, dans une certaine mesure, Marie Stritt et Marie Raschke, a ainsi initié le mouvement de protestation de femmes de plus grande ampleur jamais vue en Allemagne, avec des rassemblements publics, des collectes de signatures et des pétitions ${ }^{(7)}$. L'intervention des femmes dans l'espace public autour de cette cause a réuni pour la première fois les mouvements de femmes bourgeois et prolétaire, une union certes fragile et de courte durée. La presse allemande a rendu compte des actions collectives des femmes sur un ton amusé pour les uns, respectueux pour les autres, et le BGB a finalement été adopté en 1896 avec des amendements mineurs en faveur des femmes, pour entrer en vigueur en 1900.

\section{Les leçons de l'histoire}

Les féministes, toutes tendances confondues - excepté le mouvement prolétaire féminin, dont la leader, Clara Zetkin (1857-1933), rejetait la pétition comme l'instrument politique bourgeois par excellence -, s'étaient adressées aux législateurs en bonne et due forme et ont été ignorées. Elles ont par ailleurs dû faire le douloureux constat qu'elles ont à peine été soutenues par les députés du Reichstag, alors que ceuxci, pensaient-elles, avaient vocation à défendre les intérêts de ceux qu'ils représentent. Il fallait donc procéder autrement si les femmes voulaient voir leurs intérêts défendus.

Les féministes bourgeoises de tendance modérée ont réagi à cet état de fait en multipliant les projets fondés sur l'entraide féminine et promouvant celle-ci: ainsi, dans les «bureaux» ou, selon le cas, "associations pour la protection juridique», des femmes de tous les milieux sociaux pouvaient venir chercher un conseil juridique auprès de conseillers féminins formés à cet effet. Dans ce projet, qui a connu un succès notoire, les conseillères juridiques faisaient la promotion systématique du régime de la séparation de biens comme un moyen de réduire la dépendance financière des femmes, dans la mesure où le droit familial le permettait ${ }^{(8)}$.

Les femmes qui avaient pris la tête de la campagne pour une révision du BGB, Anita Augspurg, Marie Stritt, Marie Raschke et Minna Cauer, ont tiré d'autres leçons: puisque les décideurs politiques et les législateurs ignoraient leurs revendications, et que les représentants du peuple ne représentaient manifestement pas les intérêts des femmes, alors les femmes devaient donner la priorité à l'obtention du droit de vote. Cela leur semblait être la seule garantie de pouvoir exercer une influence sur la législation. Ainsi, le fait que la mobilisation des femmes pour les droits civils a été ostensiblement ignorée par les décideurs masculins a propulsé un certain nombre de féministes allemandes dans l'arène politique et les a poussées à concentrer leurs efforts sur le droit de vote.

7 Cf. Marie Stritt, «Rechtskämpfe», in: Helene Lange, Gertrud Bäumer (éd.), Handbuch der Frauenbewegung, t. 2, Berlin, 1901, p. 134-149.

8 Cf. Marie Stritt, «Rechtsschutz für Frauen», in: LAnge/Bäumer (éd.), Handbuch (note 7), p. 123133; Beatrix GeISEL, «Patriarchale Rechtsnormen 'unterlaufen'. Die Rechtsschutzvereine der ersten deutschen Frauenbewegung», in: U. Gerhard (éd.), Frauen (note 1), p. 683-697. 
L'étude poussée du droit familial a fait naître chez les féministes la conscience de la discrimination structurelle des femmes, et tout particulièrement des femmes mariées. Les féministes radicales ont ainsi observé qu'une femme cessait d'être un sujet de droit du jour où elle se mariait. Comment cette prise de conscience s'est-elle traduite dans le comportement marital des féministes radicales? Les féministes radicales ont-elles tourné le dos au mariage dès lors qu'elles ont pris conscience de ses conséquences sur leur statut au regard de la loi ? Un examen de leur état civil et des structures familiales dans lesquelles elles vivaient permettra d'y répondre.

\section{Les féministes radicales, toutes des vieilles filles aigries?}

L'état des sources ne permet pas d'analyser de façon systématique la biographie et le statut marital de toutes les féministes concernées. De plus, il est bien souvent malaisé de s'exprimer de façon générale sur les féministes radicales, car certaines d'entre elles, et non des moins importantes dans l'histoire du mouvement, comme Lily Gizycki (i.e. Lily Braun 1865-1916) et Käthe Schirmacher (1865-1930) ont changé d'orientation et ont quitté l'aile radicale; d'autres, comme Marie Stritt, ne se laissent pas catégoriser distinctement. Pour ces raisons, ce qui suit se restreint aux chefs de file des féministes radicales. L'étude des constellations familiales dans lesquelles elles vivaient ne révèle pas de modèle unique et confirme encore moins l'image de vieilles filles aigries et hystériques s'attaquant à l'institution du mariage en méconnaissance de la chose.

\section{Des femmes vivant ensemble}

Au-delà de Helene Lange (1848-1930) et Gertrud Bäumer (1873-1954), les deux figures de proue du mouvement féministe de tendance modérée ${ }^{(9)}$, l'exemple canonique et bien connu de femmes vivant ensemble dans les milieux féministes allemands est celui de Lida Gustava Heymann et Anita Augspurg, de dix ans son aînée, qui ont vécu et milité ensemble pendant plus de quarante ans. Avant de rencontrer Lida Gustava Heymann, Anita Augspurg vivait déjà avec une autre femme, Sophia Goudstikker (1865-1924); ensemble, elles avaient installé un atelier de photographie à Munich, qui bientôt s'était révélé une affaire tout à fait fructueuse. Käthe Schirmacher, écrivaine et journaliste, qui avait vécu des années à Paris, travaillant comme correspondante étrangère pour le mouvement féministe allemand, vivait avec son amie Clara Schleker (1852-1932), qui comme elle était engagée dans le mouvement pour le droit de vote des femmes. Dans tous les cas, la discrétion de ces femmes sur la nature de la relation qui les liait est frappante. Dans les mémoires qu'elles ont publiées en commun, Lida Gustava Heymann et Anita Augspurg se servent ainsi du terme ambigu d' "amitié» pour désigner la relation entre celle-ci et Sophia Goudstikker ${ }^{(10)}$. Käthe Schirmacher, entre-temps gagnée à l'idéologie nationaliste, recourt à des termes combinant la notion d'amitié et l'appartenance nationale, le tout avec une forte connotation masculine:

9 Angelika Schaser, Helene Lange und Gertrud Bäumer. Eine politische Lebensgemeinschaft, Cologne, Böhlau, 2000.

10 Lida Gustava Heymann, Erlebtes - Erschautes. Deutsche Frauen kämpfen für Freiheit, Recht und Frieden 1850-1940, in Zusammenarbeit mit Dr. Jur. Anita Augspurg, Meisenheim am Glan, Verlag Anton Hain, 1977, p. 15. 
«La seule chose à dire à notre sujet est: nous étions des camarades d'armes, et nous étions Prussiennes » ${ }^{(11)}$.

\section{La vie en concubinage}

L'idée que les féministes, à plus forte raison si elles étaient «radicales », n'étaient pas mariées était courante. Dans des caricatures, les féministes étaient souvent représentées comme des vieilles filles, ce qui, dans l'imaginaire collectif, était forcément dû soit à un manque de féminité, soit à un niveau d'exigences déraisonnable vis-à-vis des candidats au mariage $^{(12)}$. La féministe et pacifiste Helene Stöcker (1869-1943) a montré, en donnant l'exemple, qu'il pouvait arriver que des femmes renoncent à se marier et que cela relève d'une décision mûrement réfléchie. Elle et l'avocat berlinois Bruno Springer (1873-1931) ont en effet vécu en union libre de 1905 à $1931^{(13)}$. Pour des raisons qu'ils qualifiaient d'ordre éthique, et en dépit d'une forte pression sociale qui se faisait sentir à l'égard des hommes et, plus encore, des femmes non mariées, ils ne se sont pas mariés, considérant le mariage comme une institution surannée et superflue, et ont vécu conformément à leurs principes. Néanmoins, force est de reconnaître qu'Helene Stöcker constituait une exception parmi les femmes du mouvement féministe bourgeois et même dans son aile radicale.

\section{Femmes mariées et mères de famille}

De fait, la plupart des féministes radicales étaient mariées et avaient des enfants, exactement comme on l'attendait des femmes dans les milieux bourgeois ${ }^{(14)}$.

Louise Otto, pionnière féministe et inspiratrice des féministes radicales, était mariée à August Peters, un éditeur engagé qui a purgé une peine de sept ans de prison (18491856) pour avoir participé à la révolution de 1848; le mariage a eu lieu après sa sortie de prison, en $1858^{(15)}$. Hedwig Dohm, née Schlesinger (1831-1919), qui a davantage inspiré les féministes radicales par ses critiques acerbes de la société qu'elle ne s'est impliquée dans les actions collectives du mouvement féministe, était mariée à Ernst Dohm, éditeur en chef de l'hebdomadaire satirique Kladderadatsch, et mère de cinq enfants ${ }^{(16)}$.

À côté de femmes mariées à des éditeurs et collaborateurs de journaux critiques, d'autres féministes radicales étaient mariées à des hommes politiques: cela vaut pour les suffragistes et pacifistes Tony Breitscheid, Auguste Kirchhoff et Katharina Scheven. Tony Breitscheid, née Drevermann (1878-date de décès inconnue), était soutenue par son mari, Rudolf Breitscheid (1874-1944), libéral démocrate puis social-démocrate, dans

11 Notons qu'en allemand le genre n'est pas marqué dans la phrase «und wir waren Preußen». Käthe SCHIRMACHER, Flammen: Erinnerungen aus meinem Leben, Leipzig, Dürr \& Weber, 1921, p. 62.

12 Bärbel Kunn, Familienstand ledig. Ehelose Frauen und Männer im Bürgertum (1850-1914), Cologne/ Weimar, Böhlau, 2002.

13 Plus précisément, leur relation a débuté en 1905. Ils ont d'abord habité dans deux appartements au même étage d'une maison de Berlin-Friedenau puis décidé en 1912 de déménager pour partager un logement. Christl Wickert, Helene Stöcker 1869-1943, Frauenrechtlerin Sexualreformerin und Pazifistin. Eine Biographie, Bonn, Dietz, 1991, p. 88.

14 Sylvia Schraut, Bürgerinnen im Kaiserreich. Biografie eines Lebensstils, Stuttgart, Kohlhammer, 2013.

15 Louise Otto-Peters, in: Sächsische Biographie (online), http://saebi.isgv.de/biografie/Louise_OttoPeters_(1819-1895), consulté le 21.10.2016.

16 Isabel Rohner, Spuren ins Jetzt. Hedwig Dohm - eine Biografie, Sulzbach/Ts., Helmer, 2010. 
la lutte pour le suffrage des femmes ${ }^{(17)}$. Auguste Kirchhoff, née Zimmermann (18671940), était mariée à Heinrich Kirchhoff (1854-1929), avocat et notaire élu sénateur de la ville de Brême, avec qui elle a eu cinq enfants. Elle a soutenu la carrière professionnelle de son mari en assurant ses arrières et en assumant pleinement le rôle bourgeois d'épouse et de mère, mais lui la soutenait également, non sans quelque risque pour sa carrière, dans son engagement dans les mouvements pour la protection des mères, pour le suffrage des femmes et pour la paix ${ }^{(18)}$. Katharina Scheven, née Bauch (1861-1922), figure proéminente du mouvement abolitionniste allemand et international, a publié avec son mari Paul Scheven (1852-1929) de nombreux articles de presse, notamment dans la revue abolitionniste allemande, Der Abolitionnist. Tous deux sont devenus des acteurs centraux de la politique sociale dans la ville de Dresde et ont détenu des mandats au conseil municipal de la ville, Katharina Scheven étant l'une des premières femmes dans cette situation après $1918^{(19)}$. La suffragiste et pacifiste Margarethe Lenore Selenka, née Heinemann (1860-1922), a d'abord connu un mariage malheureux avec l'écrivain Ferdinand Neubürger. Une fois le divorce prononcé en 1893, elle a épousé le zoologue Emil Selenka (1842-1902), de 19 ans son aîné, professeur à l'université de Munich. À ses côtés, elle a acquis de solides connaissances en zoologie, qui lui ont permis d'accompagner son mari lors d'un voyage de deux ans en Asie du Sud-Est comme son assistante et de publier avec lui un ouvrage sur leur travail de terrain. En plus de ses propres recherches, Margarethe Lenore Selenka était engagée dans le mouvement féministe depuis 1897, manifestement sans opposition de la part de son mari ${ }^{(20)}$.

Ce qui est frappant dans le parcours de ces femmes, c'est qu'elles étaient toutes mariées à des représentants des professions intellectuelles: éditeurs, hommes politiques, écrivains, professeurs d'université. Leurs maris, selon toute apparence, n'étaient pas opposés à leurs activités féministes, et certains d'entre eux soutenaient même les causes qu'elles défendaient.

\section{Femmes vivant seules contre leur gré}

Emil Selenka étant décédé en 1902, Margarethe Lenore Selenka était veuve la plupart du temps de son engagement dans les mouvements féministe et pacifiste. Cela était également le cas de Minna Cauer, qui, à quarante ans, était déjà deux fois veuve. Son premier mari était médecin et le second, Eduard Cauer (1823-1881), directeur de Gymnasium et inspecteur académique. C'est lui qui avait incité sa femme à rejoindre le mouvement féministe alors à peine constitué, lui ramenant à la maison les Neue Bahnen (Nouvelles voies), l'organe de presse de l'ADF. Dans ses journaux intimes, Minna Cauer soulignait tous les ans la date anniversaire du décès d'Eduard Cauer, en qui elle voyait

17 Anne-Laure Briatte-Peters, Citoyennes sous tutelle. Le mouvement féministe «radical» dans l'Allemagne wilhelmienne, Berne, Peter Lang, 2013.

18 Auguste KIRchноғ, «Mensch sein, heißt Kämpfer sein!» Schriften für Mutterschutz, Frauenrechte, Frieden und Freiheit 1914-1933, Brême, Donat, 2004, p. 10.

19 Barbara Hillen, «Scheven, Katharina», in: Neue Deutsche Biographie, 22 (2005), p. 717 sq. (online), https://www.deutsche-biographie.de/gnd117621668.html\#ndbcontent (19.10.2016).

20 Ute Kätzel, «A Radical Women’s Rights and Peace Activist: Margarethe Lenore Selenka, Initiator of the First Worldwide Women's Peace Demonstration in 1899 », Journal of Women's History, 13/3 (2001), p. 46-69. 
une âme sœur, que nul(le) autre n'a pu remplacer par la suite. Alice Salomon (18721948), pionnière du travail social, ne prétendait pas représenter le mouvement féministe radical, mais c'est bien là qu'a commencé sa carrière féministe avant de se poursuivre dans des associations et des actions de nature plus modérée. Dans son autobiographie, elle explique pourquoi elle ne s'est jamais mariée: non qu'elle ait rejeté le mariage par principe, mais elle n'a pas pu obtenir les hommes qui l'intéressaient et n'était pas intéressée par ceux qu'elle aurait pu avoir. Elle commente: «Voilà toute la vérité. Les femmes ne décident pas de ces choses sur la base d'un principe ou d'une théorie »(21).

L'indépendance sociale, si elle allait de pair avec une indépendance financière, était sans conteste une condition favorable à l'engagement féministe, qui demandait du temps, des déplacements et des moyens. Mais plusieurs figures importantes parmi les féministes radicales avaient des obligations familiales. Il n'était pas rare, dans ce cas, que les femmes s'engagent une fois que les enfants avaient grandi; c'est ce qu'avait fait Marie Stritt, épouse d'un chanteur d'opéra, mère de deux enfants et figure de proue du mouvement féministe bourgeois dans les années 1900-1910 ${ }^{(22)}$. En fin de compte, ce que les féministes radicales, mariées ou non, avec ou sans charges familiales, avaient en commun, était la jouissance d'une liberté individuelle dans leur environnement proche et, pour les femmes mariées, un soutien direct ou indirect de leur conjoint. Ces dernières en particulier pouvaient en outre généralement tirer profit de la position sociale et s'appuyer sur les réseaux de leurs maris.

\section{Amour et responsabilité au fondement d'une nouvelle éthique}

Un sens très aigu de la liberté: telle pourrait être une façon de caractériser les féministes radicales. Très attachées au principe libéral, hérité des Lumières, du libre développement des facultés de l'individu, elles accordaient une grande importance à la liberté individuelle. Celle-ci était pour elles à l'opposé d'une recherche hédoniste du plaisir : conformément à la morale kantienne, dans laquelle elles avaient grandi, la liberté était intrinsèquement liée à un sens du devoir (Pflicht $)$. Si les féministes radicales allemandes critiquaient l'institution du mariage, elles le faisaient parce que le code civil et les normes sociales en vigueur empêchaient les femmes d'agir librement et d'endosser des responsabilités, d'où émane la liberté. L'appel au boycott du mariage lancé par Anita Augspurg visait à attirer l'attention de l'opinion publique sur les effets dévastateurs du mariage sur la capacité d'action et l'estime de soi des femmes.

\section{Appel au boycott du mariage}

Première Allemande à obtenir le titre de docteur en sciences juridiques (en 1897), Anita Augspurg s'était imposée comme une spécialiste des questions juridiques, en particulier du droit familial. En 1905, une jeune femme gagnant sa vie s'est adressée à elle par courrier pour lui demander conseil: elle et l'homme qu'elle aimait devaientils se marier ou devaient-ils vivre en concubinage pour rester des individus égaux, et

21 Alice Salomon, Lebenserinnerungen. Jugendjahre-Sozialreform-Frauenbewegung-Exil, Francfort, Brandes \& Apsel, 2008, p. 63.

22 Elke Schüller, Marie Stritt, eine «kampfrohe Streiterin» in der Frauenbewegung (1855-1928), Königstein, Helmer, 2005. 
ce en dépit du prix à payer, sur le plan social et professionnel, pour un tel mode de vie? Voyant dans ce dilemme un "cas typique du temps présent», Anita Augspurg lui a répondu dans une lettre ouverte d'abord publiée dans le magazine Europe puis, quelques semaines plus tard, dans Die Frauenbewegung, l'organe de presse officiel du mouvement féministe radical $^{(23)}$. Sa réponse se résume dans la phrase suivante:

«Pour une femme ayant l'estime d'elle-même et la connaissance des effets d'une union maritale au regard de la loi, il est à mon avis impossible de se marier légalement: son instinct de conservation, le respect de sa personne et son droit d'attendre le respect de son mari ne lui laissent d'autre choix que l'union libre ${ }^{(24)}$.

La lettre ouverte d'Anita Augspurg s'attaquait à l'institution du mariage, pilier de la société bourgeoise, ce qui n’a pas manqué de susciter de vifs débats non seulement dans la société, mais aussi au sein du mouvement féministe allemand.

\section{Le concept d'union libre selon Helene Stöcker}

Helene Stöcker a joué un rôle considérable dans le débat sur le mariage qui s'étendait à des cercles de plus en plus étendus, en proposant une réforme complète de la «morale sexuelle», c'est-à-dire des normes relatives à la sexualité et de la morale en général. Par divers moyens, elle s'est efforcée, des décennies durant, de faire valoir sa conception d'une "Nouvelle éthique », qui, à ses yeux, devait s'imposer pour le progrès de l'humanité. Outre ses nombreux articles publiés et des tournées de conférences à travers tout l'Empire, Helene Stöcker entendait diffuser ses idées et favoriser les discussions autour d'une réforme de la morale sexuelle avec la création de l'Alliance pour la protection des mères et pour une réforme sexuelle (Bund für Mutterschutz und Sexualreform), avec d'autres réformateurs, médecins et féministes à Berlin en 1905.

Helene Stöcker ne cherchait pas spécialement à abolir le mariage, mais elle aspirait à ce que le corps et l'âme soient réunis dans la vie sexuelle d'un couple, ce qui selon elle n'était pas possible dans la «morale surannée des temps présents ${ }^{(25)}$. Dans son idéal d'amour - amour qu'elle ne concevait qu'hétérosexuel, à l'exclusion d'autres formes de sexualités -, l'homme et la femme se respecteraient mutuellement, étant tous deux conscients de l'égale valeur des deux sexes. Pour parvenir à ce stade de maturité personnelle, il fallait que les jeunes gens reçoivent une éducation commune, en «co-éducation», comme cela se pratiquait encore trop rarement dans les écoles ${ }^{(26)}$. Helene Stöcker définissait sa «Nouvelle éthique» comme «une croyance dans l'éthique de la responsabilité personnelle, en particulier dans le champ sexuel» ${ }^{(27)}$. En référence implicite à l'impératif catégorique chez Emmanuel Kant, elle affirmait:

23 Anita Augspurg, "Ein typischer Fall der Gegenwart. Offener Brief», Europa, 27.04.1905, p. 311-314, repr. Die Frauenbewegung, 01.06.1905, p. 81 sq.

24 Ibid., p. 81.

25 Helene Stöcker, «Das Werden der sexualen Reform seit hundert Jahren », in: Hedwig Doнm, Anita Augspurg, Helene Stöcker et al. (éd.), Ehe? Zur Reform der sexuellen Moral, Berlin, 1911, p. 38-41.

26 Rebecca Rogers, "L'enseignement mixte en Europe (XIX $\mathrm{XXXI}^{\mathrm{e}}$ siècles) », in: Encyclopédie pour une histoire nouvelle de l'Europe (en ligne), 2016, mis en ligne le 24.06.2016, consulté le 20.10.2016, ehne.fr/ article/genre-et-europe/eduquer-des-europeens-et-des-europeennes/lenseignement-mixte-eneurope-xixe-xxie-siecles.

27 H. Stöcker, «Das Werden» (note 25), p. 38. 
«La personnalité responsable, que ce soit d'un homme ou d'une femme, est au centre de la Nouvelle éthique. Elle doit devenir aussi le centre de la loi moderne» ${ }^{(28)}$. Ainsi, son éthique du couple reposait sur deux piliers: l'amour et la responsabilité, l'amour étant défini comme la combinaison de l'affinité mentale et du désir sexuel ${ }^{(29)}$. Car l'un des enjeux au cœur de sa lutte pour une réforme sexuelle était de réhabiliter le désir sexuel pour les deux sexes. Cela précisément lui a valu de nombreuses attaques dirigées contre elle, qu'on suspectait de menacer l'institution de la famille et, par suite, la société dans son entier.

\section{Controverse sur le mariage au sein du mouvement féministe à la veille de la guerre}

La vision stöckerienne d'une Nouvelle éthique fondée sur l'amour et la responsabilité entre deux individus libres qui, étant donné le droit familial en vigueur, ne pouvaient que faire le choix de l'union libre (freie Ehe, littér. le mariage libre), ne faisait pas l'unanimité, loin s'en faut. Ses adversaires confondaient le plus souvent l'union libre (freie Ehe) avec le libertinage amoureux (freie Liebe), ou feignaient de les confondre pour pouvoir plus aisément jeter l'opprobre sur le camp adverse. Ainsi, dans ses mémoires, Alice Salomon, qui pourtant avait une position assez critique sur la conception dominante du mariage, confond systématiquement l'union libre et le libertinage ${ }^{(30)}$. Ce faisant, elle a contribué avec d'autres à faire croire que Helene Stöcker encourageait la dissolution des mœurs, une réputation dont l'intéressée a eu toutes les peines à se défaire ${ }^{(31)}$.

De fait, la plupart des féministes allemandes désapprouvaient les unions hors mariage. La «critique du mariage» (Ehekritik), comme a été appelée la controverse autour du mariage dans les années d'avant-guerre, a divisé le mouvement féministe bourgeois allemand en deux camps. D’un côté, la plupart des féministes modérées préconisaient une réforme juridique du mariage, dont elles espéraient l'abolition de toute espèce d'hégémonie patriarcale et, par suite, la consolidation du mariage, qui constituait selon elles «l'objectif suprême» ${ }^{(32)}$. À leurs yeux, le concubinage «sap[ait] le mariage et la famille» ${ }^{(33)}$. De l'autre côté, la plupart des féministes radicales et des réformateurs progressistes préconisaient une libéralisation des normes morales qui rendrait possible une vie sexuelle en dehors du cadre du mariage et qui seule permettrait l'émergence d'une société libre faite d'individus responsables. Les adversaires et les défenseurs du mariage s'affrontaient ainsi dans une "féroce bataille de principes » ${ }^{(34)}$, jusqu’à ce que la guerre éclate, faisant tourner court ce débat essentiel.

L'unification des codes civils allemands dans le BGB, entreprise à une époque de transformations sociales qui ne pouvaient pas ne pas affecter les rapports hommesfemmes, a consolidé, pour ne pas dire verrouillé, l'hégémonie masculine dans la

28 Ibid., p. 40.

29 Ibid., p. 56.

30 A. SALOMON, Lebenserinnerungen (note 21), p. 147 sq.

31 H. Stöcker, «Das Werden» (note 25), p. 58.

32 Gertrud Bäumer, Dr. Med. Agnes Bluhm, Ika Freudenberg et al. (éd.), Frauenbewegung und Sexualethik. Beiträge zur modernen Ehekritik, Heilbronn, Eugen Salzer, 1909, p. VII.

33 Ibid.

34 Ibid. 
famille et la société. Ignorées et renvoyées à leur rôle domestique, les femmes qui ont tenté d'intervenir dans ce processus dont elles avaient très bien perçu l'enjeu ont pris conscience du caractère structurel de la subordination des femmes et du projet politique d'une hiérarchie des genres qu'elle servait. C'est précisément cette prise de conscience et leur défaite dans la lutte contre le BGB qui ont fait émerger une aile «radicale» dans le mouvement féministe bourgeois. Les féministes radicales allemandes n'étaient pas pour autant de farouches ennemies du mariage. Seule une minorité d'entre elles était opposée au mariage par principe et a choisi - et assumé dans toutes ses conséquences un autre mode de vie. Leurs actions collectives et les modèles de vie alternatifs qu'elles proposaient ont déchaîné de vifs débats dans l'opinion publique. Au-delà des polémiques, la qualité et la vivacité de ces débats peuvent être interprétées comme l'indice d'une ouverture possible de la société allemande, un processus que le déclenchement de la guerre a interrompu brusquement.

\section{Résumé}

Cette contribution se penche sur la critique de la famille, du mariage et de la sexualité formulée par les féministes radicales du mouvement féministe bourgeois dans l'Allemagne wilhelmienne. L'analyse s'ouvre sur l'impact de l'unification du code civil allemand sur le droit familial et conjugal et les tentatives échouées de féministes d'influer sur cette codification. Une présentation des modèles familiaux dans lesquels vivent les féministes radicales montre que la liberté dans le couple à laquelle elles aspiraient n'était pas la recherche hédoniste du plaisir. Des initiatives comme le "boycott du mariage» et la controverse entre les féministes sur le mariage permettent finalement de comprendre que les radicales conçoivent la liberté dans le couple comme fondée sur le respect de la personne et la responsabilité des individus. Elles voient là les conditions nécessaires à l'avènement d'une société d'individus libres et responsables.

\section{Zusammenfassung}

Dieser Beitrag ist der Kritik der Familie, der Ehe sowie der Sexualmoral durch die radikalen Frauenrechtlerinnen der bürgerlichen Frauenbewegung im Deutschen Kaiserreich gewidmet. Zuerst untersucht er die Auswirkungen der Vereinheitlichung des deutschen Zivilrechts auf das Familien- und Eherecht und die gescheiterten Versuche von Frauenrechtlerinnen, auf diese Kodifikation Einfluss zu nehmen. Eine Übersicht der Familienkonstellationen, in denen die radikalen Frauenrechtlerinnen lebten, zeigt, dass die Freiheit in der Liebe, die sie anstrebten, nichts mit einer hedonistischen Suche nach sexueller Lust zu tun hatte. Durch Initiativen wie der „Eheboykott" und die Kontroverse über die Ehe unter den Frauenrechtlerinnen wird klar, dass die radikalen Frauenrechtlerinnen die Freiheit in der Liebe in der Achtung der Person und der Verantwortung des Individuums begründet wissen wollten. Darin sahen sie die notwendigen Voraussetzungen für eine Gesellschaft von freien und verantwortlichen Menschen. 